\title{
Neurotoxicity of paraquat and paraquat-induced mechanisms of developing Parkinson's disease
}

Laboratory Investigation (2016) 96, 1028-1029; doi:10.1038/labinvest.2016.80

Sir

We read with some interest the 'Mini Review' article by Zhang, Thompson and Xu entitled 'Multifactorial theory applied to the neurotoxicity of paraquat and paraquatinduced mechanisms of developing Parkinson's disease' recently published in Laboratory Investigation. ${ }^{1}$ Although we found the mini review proposed an interesting theory that may add to the debate around the aetiology of idiopathic Parkinson's disease, we would like to seek the opportunity to point out to the readership of Laboratory Investigation some relevant shortcomings relating to this article with respect to the apparent omission by the authors of highly relevant papers authored or commissioned by ourselves, which seriously question the veracity and reliability of the paraquat animal model studies frequently referred to in the mini review, as well as human epidemiological studies which cast doubt on the claimed association between paraquat exposure and the incidence of Parkinson's disease. In addition, the article includes a number of misconceptions and incorrect statements made by the authors with respect to paraquat, its use in crop protection and both environmental and human exposure.

We consider it is particularly important to make these comments, not only because the omissions impact on the general conclusions reached by the authors regarding the potential neurotoxicity of paraquat and its relationship to the aetiology of Parkinson's disease, but also the incorrect statements relating to paraquat could mislead some readers, and we would wish to avoid the same errors being repeated in subsequent publications by readers of this article.

The authors state that they reviewed the environmental context and biochemistry of paraquat in soils, water and organisms to identify potential routes that can lead to chronic rates of low-dose exposure which would replicate the type of response that is observed in animal models, epidemiological studies and other types of laboratory investigations involving paraquat exposure. However, the authors neglected to mention that there are published studies using animal models, including a 13-week dietary study (Minnema et $\left.a l^{2}\right)$, and in humans, involving paraquat manufacturing workers (Tomenson and Campbell ${ }^{3}$ ), clearly demonstrating that chronic low-dose exposure to paraquat is without effect. In fact, some of the animal model studies referred to by the authors to support their assertions were neither low-dose or chronic in nature, since they involved the administration of high doses to rodents (typically $>1 / 3 \mathrm{rd}$ of the median lethal dose) for relatively short periods (up to three weeks) by means of weekly intraperitoneal (ip) injection, a dose route which has no relevance to any normal human exposure scenario. Furthermore, there is no mention by the authors regarding the robustness and reproducibility of the animal models which have been seriously called into question by the failure of some research groups to replicate the previously reported neurotoxicity in the ip paraquat mouse model (Breckenridge et al ${ }^{4}$ ) and which has been further complicated by at least one known instance of scientific fraud (US Federal Register Notice ${ }^{5}$ ). These studies, which were omitted by the authors in their review of the current literature, clearly undermine the authors' assertions linking Parkinson's disease to paraquat exposure.

The epidemiological evidence relating to a causal relationship between paraquat use or exposure and Parkinson's disease suffers from a number of limitations relating to the accuracy of exposure measurements. In contrast to the statements made by the authors, a higher incidence of Parkinson's disease development in areas subject to paraquat exposure has not been reliably demonstrated and systematic review meta-analysis (Mandel $e t a l^{6}$ ) and weight of evidence assessments (Breckenridge $e t a l^{7}$ ) do not allow one to infer a causal relationship between paraquat exposure and Parkinson's disease. We would agree that a more rigorous and robust approach to the epidemiological studies underlying such meta-analyses is currently required to gain further understanding in this regard. Two epidemiological studies (not mentioned in the authors' review) where two groups of humans known to be (or likely to be) substantially exposed to paraquat have revealed no causal link to Parkinson's disease. Brent and Schaeffer ${ }^{8}$ assessed whether confirmed high-dose exposure to paraquat (as a result of oral ingestion) was associated with the subsequent development of Parkinsonism 
- and there was no connection. Tomenson and Campbell ${ }^{3}$ concluded that there was no evidence of an increased incidence of Parkinson's disease among a cohort of paraquat production workers with prolonged relatively high exposure to paraquat. The strengths of both these studies lie in the known exposure to paraquat, rather than proxy or selfreported exposure based upon responses to questionnaires, often recorded long after any presumed exposure may have occurred.

The authors state that 'there are multiple avenues for chronic low-dose exposure of $P Q$ through the environmental context', however this is fundamentally incorrect and misleading. Paraquat, of all crop protection chemicals, is not likely to result in chronic low-dose exposure through the environment as on contact with soil it is rapidly bound and becomes biologically deactivated (Roberts, Dyson and Lane, ${ }^{9}$ Lane), it does not leach into groundwater (US EPA RED ${ }^{10}$ ) and uses do not typically result in appreciable residues in the edible portion of the crop. Most uses for paraquat involve spraying weeds and not crops. Because the paraquat is biologically deactivated on contact with the soil, it cannot be taken up by plant roots. Consequently, for the vast majority of uses, no residues are expected in harvested crops. This has been confirmed over many years by analysis of crops harvested after the use of paraquat for weed control. There are a few situations where crops are treated directly when paraquat is used as a pre-harvest desiccant. These uses are approved by regulatory authorities who have confirmed that such treatments do not leave residues at levels which represent a risk to humans. References to any appreciable concentration of paraquat being detected in ground and surface water are completely implausible based on the known physico-chemical profile of paraquat. However, it is possible that in such occasionally reported cases the sampling was of sedimentbound, but biologically deactivated paraquat. In addition, statements relating to the risk of trophic accumulation of paraquat owing to its accumulation in animal tissues are equally implausible, since paraquat does not bio-accumulate. On this basis, any human dietary exposure to paraquat is negligible and there is no exposure via drinking water. In this context, any proposals to test farm animals or captured wild animals make no sense, since there is no expectation of any significant exposure and no means of measuring past exposure. Neither is there a clear understanding of what, precisely, one would be looking for in relation to Parkinson's disease.

Finally, there are two further statements contained within the article which are not true and we seek, as a matter of record, to correct them:

'Organophosphates, which can be combined with PQ (eg, organophosphate PQ is produced commercially Gramoxone), are also implicated in increasing risk of PD'.
Organophosphates are NOT combined with paraquat in 'Gramoxone'. There is absolutely no agronomic rationale for such a combination, that is, an organophosphate insecticide and a non-selective herbicide.

'PQ is banned in the European Union since 2007.'

Paraquat is NOT banned in the European Union (EU). A July 2007 EU Court decision revoked paraquat registrations, finding that the EU Commission's review failed to satisfy certain administrative and procedural requirements. At no stage did the Court conclude that paraquat was an inherently unsafe or dangerous product and should be 'banned'. The main registrant of paraquat, Syngenta, decided not to pursue a re-submission for the registration of paraquat in Europe, since the re-submission would require significant expenditure to conduct what it considered unnecessary further regulatory studies. These costs outweighed the product's market opportunities in the region.

Andrew R Cook ${ }^{1}$, Philip A Botham ${ }^{1}$, Charles B Breckenridge ${ }^{2}$, Daniel J Minnema ${ }^{2}$, Nicholas C Sturgess ${ }^{1}$ and Kim Z Travis ${ }^{1}$ ${ }^{1}$ Syngenta Limited, Jealott's Hill International Research Centre, Bracknell, Berkshire, UK and ${ }^{2}$ Syngenta Crop Protection, LLC, Greensboro, NC, USA

E-mail:andy.cook@syngenta.com

\section{DISCLOSURE/CONFLICT OF INTEREST}

All authors of this letter are current employees of Syngenta Crop Protection, LLC or Syngenta Limited.

1. Zhang $\mathrm{XF}$, Thompson $\mathrm{M}, \mathrm{Xu} \mathrm{YH}$. Multifactorial theory applied to the neurotoxicity of paraquat and paraquat-induced mechanisms of developing Parkinson's disease. Lab Invest 2016;96:496-507.

2. Minnema DJ, Travis KZ, Breckenridge CB, et al. Dietary administration of paraquat for 13 weeks does not result in a loss of dopaminergic neurons in the substantia nigra of C57BL/6 J mice. Regul Toxicol Pharmacol 2014;68:250-258.

3. Tomenson JA, Campbell C. Mortality from Parkinson's disease and other causes among a workforce manufacturing paraquat: a retrospective cohort study. BMJ Open 2011;1:e000283.

4. Breckenridge $C B$, Sturgess $N C$, Butt $M$, et al. Pharmacokinetic neurochemical, stereological and neuropathological studies on the potential effects of paraquat in the substantia nigra pars compacta and striatum of male C57BL/6 J mice. Neurotoxicology 2013;37:1-14.

5. US Federal Register Notice Volume 77, 2012, 38632-38633; available from http://www.gpo.gov/fdsys/pkg/FR-2012-06-28/html/2012-15887. htm.

6. Mandel JS, Adami H-O, Cole P. Paraquat and Parkinson's disease: an overview of the epidemiology and a review of two recent studies. Regul Toxicol Pharmacol 2012;62:385-392.

7. Breckenridge $C B$, Berry $C$, Chang ET, et al. Association between Parkinson's disease and cigarette smoking, rural living, well-water consumption, farming and pesticide use: systematic review and metaanalysis. PLoS ONE 2016;11:1-42.

8. Brent J, Schaeffer TH. Systematic review of Parkinsonian syndromes in short- and long-term survivors of paraquat poisoning. J Occup Environ Med 2011;53:1332-1336.

9. Roberts TR, Dyson JS, Lane MCG. Deactivation of the biological activity of paraquat in the soil environment: a review of long-term environmental fate. J Agric Food Chem 2002;50:3623-3631.

10. US EPA Re-registration Eligibility Document. RED 1997. 